\title{
Effects of Fasting on Plasma Lipoprotein(a) in Cynomolgus Monkeys: Preliminary Experiments Results
}

\author{
Masataka Kusunoki ${ }^{*}$, Kazuhiko Tsutsumi², Daisuke Sato ${ }^{3}$, Tetsuro Miyata4, \\ Takao Nakamura ${ }^{3}$ \\ ${ }^{1}$ Department of Internal Medicine, Medical Clinic, Aichi Medical University, Nagoya, Japan \\ ${ }^{2}$ Okinaka Memorial Institute for Medical Research, Tokyo, Japan \\ ${ }^{3}$ Department of Biomedical Information Engineering, Graduate School of Medical Science, Yamagata University, \\ Yamagata, Japan \\ ${ }^{4}$ Vascular Center, Sanno Medical Center, Tokyo, Japan \\ Email: info@tonyo.jp
}

Received 30 June 2014; revised 17 August 2014; accepted 5 September 2014

Copyright (C) 2014 by authors and Scientific Research Publishing Inc.

This work is licensed under the Creative Commons Attribution International License (CC BY).

http://creativecommons.org/licenses/by/4.0/

(c) (i) Open Access

\section{Abstract}

Plasma lipoprotein(a) (Lp(a)) is known to be a strong independent risk factor for ischemic heart disease. However, it is not easily modulated by drugs that are presently available. Lp(a) is not present in many experimental animals except for Old World monkeys. In this study, we examined whether cynomolgus monkeys are useful model for research of human plasma Lp(a), and observed that plasma Lp(a) in cynomolgus monkeys varied as much as humans. As a result of 4 day-fasting in cynomolgus monkeys, the plasma Lp(a) level decreased in a monkey with originally high Lp(a) level. The Lp(a) level continued to decrease even 3 days after banana feeding, but returned to the original level 3 days after monkey chow feeding. On the other hand, in a monkey with low Lp(a) level, fasting had no effect on the Lp(a) level. However, in the third monkey having originally high Lp(a) level, the Lp(a) was not affected by decreasing the amount of monkey chow feeding by $50 \%$. In summary, we found that cynomolgus monkeys may be an useful model for studying the effects of food on plasma Lp(a) in place of humans, and that high Lp(a) level may be controllable by strict diet regulation.

\section{Keywords}

Lipoprotein(a), Fasting, Cynomolgus Monkey, Banana

\footnotetext{
*Corresponding author.

How to cite this paper: Kusunoki, M., Tsutsumi, K., Sato, D., Miyata, T. and Nakamura, T. (2014) Effects of Fasting on Plasma Lipoprotein(a) in Cynomolgus Monkeys: Preliminary Experiments Results. Open Journal of Animal Sciences, 4, $222-227$. http://dx.doi.org/10.4236/ojas.2014.45028
} 


\section{Introduction}

Apolipoprotein(a) is a glycoprotein that attaches to apolipoprotein B (apoB) in plasma low-density lipoprotein (LDL) particles, forming lipoprotein(a) (Lp(a)) [1] [2]. Several studies have demonstrated a strong association between $\operatorname{Lp}(\mathrm{a})$ and cardiovascular disease. It has also been reported that there is a strong positive relationship between elevated plasma levels of $\mathrm{Lp}(\mathrm{a})$ and myocardial infarction as well as angiographically-defined coronary artery disease [1] [3]. The importance of decreasing elevated plasma $L p(a)$ levels depends on whether a reduction in plasma Lp(a) levels will be accompanied by a reduction in the risk of cardiovascular disease due to elevated Lp(a) levels. However, Lp(a) levels are not easily modulated by diet or by routinely employed medications [4].

Recently, it have been reported that dimyristoylphosphatidylcholine inhibits Lp(a) formation in transgenic mice [5] (Wang et al., 2009), and that existing anti-rheumatic agents reduces serum Lp(a) levels in rheumatic patients [6] [7]. We hope to find a novel drug that reduces $L p(a)$ and determine a food treatment that regulates $\mathrm{Lp}(\mathrm{a})$ levels. However, $\mathrm{Lp}(\mathrm{a})$ is not present in many species of experimental animals for researching Lp(a) except for Old World monkeys (e.g., cynomolgus monkey and rhesus monkey) [8] [9] and Lp(a) transgenic animals [5] [10].

In this study, we preliminarily investigated that whether cynomolgus monkeys are possible to use for research of Lp(a) instead of humans, and determined the effects of fasting on plasma Lp(a) levels in cynomolgus monkeys.

\section{Materials and Methods}

\subsection{Animals}

Three Philippine male cynomolgus monkeys weighing approximately $5.8 \mathrm{~kg}$ and aged 5 to 8 years old were obtained from Keari Co., Ltd. (Wakayama, Japan). They were housed in stainless steel cages $(100 \mathrm{~W} \times 100 \mathrm{D} \times$ $165 \mathrm{H} \mathrm{cm}$, CLEA Japan, Inc.) in conventional housing. The trays under the cages were changed once a day. Room temperature was maintained at $23^{\circ} \mathrm{C} \pm 2{ }^{\circ} \mathrm{C}$ with $55 \% \pm 10 \%$ relative humidity. The artificial light/dark cycle was 12 hours with lights on at 7:00 am, and there was an average of 13 - 16 air changes per room per hour. Monkeys were fed 150 g of monkey chow (Monkey BIT, Nihon Nosan Kogyo Co., Ltd., Yokohama, Japan) once a day at 10:00 am. The monkey chow consisted of 9.0\% water, 20.5\% protein, 8.5\% fat, $4.0 \%$ fiber, $7.4 \%$ ash and 50.6\% carbohydrate. Metabolic energy was $303 \mathrm{kcal}$ per $100 \mathrm{~g}$ of the monkey chow. All animals had access to tap water ad libitum.

All animal experiments were approved by the animal ethics committee of Aichi Medical University and conformed with the Guide for the Care and Use of Laboratory Animals issued by the National Institutes of Health.

\subsection{Animal Experiments}

Flow chart of this study is shown in Figure 1. After a standard monkey chow feeding, two monkeys (one with a high level of $\mathrm{Lp}(\mathrm{a})$ and one with a low level of $\mathrm{Lp}(\mathrm{a})$ ) were placed on a zero diet for 4 days. After the fasting, both monkeys received one banana (approximately $100 \mathrm{~g}$ )/day for 3 days. And then, the monkeys received 150 g/day of the monkey chow for 3 days.

The third monkey having a high level of $\mathrm{Lp}(\mathrm{a})$ was placed on a diet consisting of half the amount of monkey chow fed per day (75 g) to determine whether mild affects Lp(a) levels.

Blood samples were obtained from an antebrachial vein before the start of the experiment, and 4, 7 and 10 days after the start of experiment at 9:00 am for determination of plasma lipids using a heparinized syringe.

\section{Results}

Plasma lipid levels are shown in Table 1. Regarding pre-fasting Lp(a) levels, monkey no. 3 had the highest level of Lp(a), monkey no. 1 also had a high Lp(a) value, but was lower than monkey no. 3, monkey no. 2 had the lowest level. Plasma Lp(a) levels varied among the monkeys.

In monkey no. 1 with an originally high Lp(a) level, the Lp(a) level decreased 26\% after fasting for 4 days.

This reduction reached $40 \%$ after 3 days of banana feeding. The $\mathrm{Lp}(\mathrm{a})$ level recovered to the original level at day 10, after 3 days of the monkey chow feeding. The TC and HDL-C levels were not affected by fasting for 4 


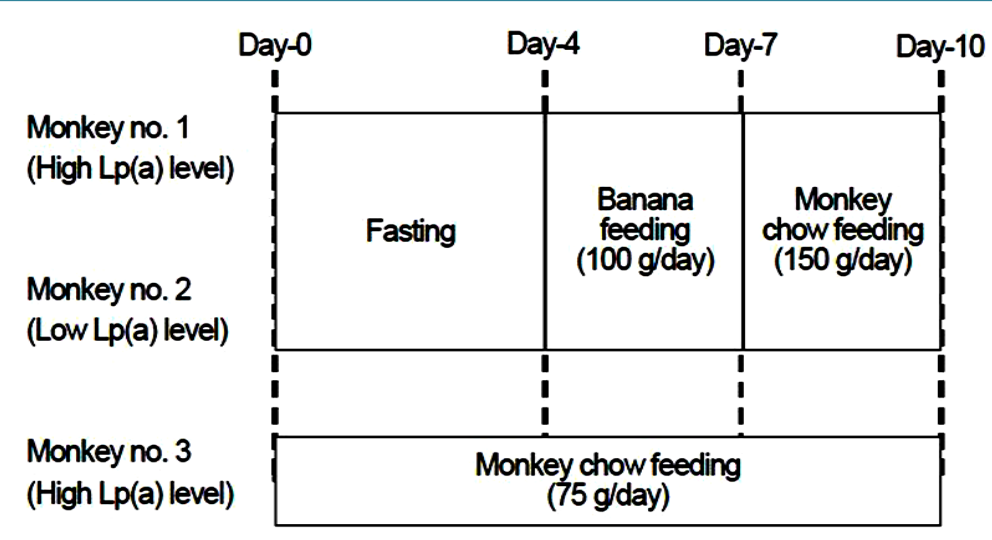

Figure 1. Schema of this study. Until start of the experiment period, all three monkeys were fed with a standard monkey chow (150 g/day).

Table 1. Plasmalipid levels in cynomolgus monkeys.

\begin{tabular}{|c|c|c|c|c|c|}
\hline Animal Number & & Before & 4 days & 7 days & 10 days \\
\hline \multirow[t]{5}{*}{1} & Lp(a) (mg/dl) & 31 & 23 & 19 & 32 \\
\hline & TC (mg/dl) & 92 & 90 & 69 & 95 \\
\hline & HDL-C (mg/dl) & 51 & 52 & 31 & 49 \\
\hline & TG (mg/dl) & 35 & 56 & 60 & 37 \\
\hline & $\operatorname{NEFA}(\mu \mathrm{Eq} / \mathrm{l})$ & 275 & 2530 & 324 & 288 \\
\hline \multirow[t]{5}{*}{2} & Lp(a) (mg/dl) & 14 & 15 & 13 & 16 \\
\hline & TC (mg/dl) & 85 & 68 & 65 & 81 \\
\hline & HDL-C (mg/dl) & 43 & 35 & 34 & 41 \\
\hline & TG (mg/dl) & 48 & 62 & 67 & 50 \\
\hline & $\operatorname{NEFA}(\mu \mathrm{Eq} / \mathrm{l})$ & 350 & 2730 & 386 & 325 \\
\hline \multirow[t]{5}{*}{3} & Lp(a) (mg/dl) & 42 & 45 & 43 & 41 \\
\hline & TC (mg/dl) & 96 & 94 & 89 & 99 \\
\hline & HDL-C (mg/dl) & 53 & 51 & 52 & 54 \\
\hline & TG (mg/dl) & 45 & 48 & 45 & 46 \\
\hline & $\operatorname{NEFA}(\mu \mathrm{Eq} / 1)$ & 326 & 365 & 355 & 334 \\
\hline
\end{tabular}

Lp(a), lipoprotein(a); TC, plasma total cholesterol; HDL-C, plasma high density lipoprotein cholesterol; TG, plasma triglyceride; NEFA, plasma nonesterified fatty acid.

days, but decreased $25 \%$ and $40 \%$, respectively, after 3 days of banana feeding. The TC and HDL-C levels increased after 3 days of the monkey chow feeding. The TG level was increased by fasting, and further increased after banana feeding, but returned to the original level after the monkey chow feeding for 3 days. The NEFA level increased 9.2-fold after 4 days of fasting, respectively. However, NEFA levels decreased after banana and monkey chow feeding.

In monkey no. 2 with an originally low level of $\mathrm{Lp}(\mathrm{a})$, the $\mathrm{Lp}(\mathrm{a})$ level did not decrease after fasting. However, the TC and HDL-C levels decreased and the TG and the NEFA levels increased after fasting. The NEFA level returned to the original level after banana feeding. The TC, HDL-C and TG levels returned to near original values after the monkey chow feeding.

In monkey no. 3, halving the amount of monkey chow fed showed almost no effect on plasma lipid levels. 


\section{Discussion}

Lp(a), which was originally discovered in 1963, has recently been confirmed to be atherogenic [11]. Plasma Lp(a) levels seem to be largely regulated by genetic factors [4] [8] [12]. There are large individual difference of Lp(a) levels in human. Accordingly, it is thought that these levels are not always affected by drug treatment or diet. In this study, we used cynomolgus monkeys which react with anti-human Lp(a) sera [13]. We found that plasma Lp(a) levels of cynomolgus monkeys showed large individual variation, similar to humans.

Recently, it has been reported that the novel apoB synthesis inhibitor, mipomersen, potentially reduced Lp(a) level in patients with familial hypercholesterolemia and coronary artery disease [14]. So far there has been many papers reporting the effects of lipid-lowering drugs on plasma Lp(a) in human. However, at least current lipid-lowering drugs other than nicotinic acid and it’s derivative did not have a reducing effect on plasma Lp(a) [8] [12].

There are also reports mentioning the effects of dietary fatty acid on plasma Lp(a) level. In a part of the reports, it is described that especially n-3 polyunsaturated fatty acid has a mild Lp(a)-reducing effect [4]. In contrast, Kooshki et al. reported that n-3 fatty acids did not affect serum Lp(a) level in human [11]. It seemed that consensus on the effects of dietary fatty acid on $\mathrm{Lp}(\mathrm{a})$ has not yet been formed. Furthermore, very few studies have dealt with the effects of fasting on plasma $\operatorname{Lp}(\mathrm{a})$.

We studied whether fasting had a reducing effect on plasma Lp(a) levels by using cynomolgus monkeys. In this study, the plasma Lp(a) level decreased after fasting for 4 days in a monkey with an originally high level of $\mathrm{Lp}(\mathrm{a})$, but not in a monkey with an originally low level of $\mathrm{Lp}(\mathrm{a})$. The plasma $\mathrm{Lp}(\mathrm{a})$ level of the latter at the beginning of the experiment was less than half the level of the former (Table 1). These results may indicate that in monkeys with originally high levels, fasting results in a decrease in Lp(a) levels, but not in monkeys with originally low levels of Lp(a). NEFA levels in monkey no. 1 and 2 were markedly increased. It is well known that fasting cause elevation of NEFA level in order to produce energy sauce through fatty acid oxydation.

Kostner et al. have reported that five obese patients were placed on a zero diet for 3 weeks, but Lp(a) levels did not change [15]. Mean Lp(a) levels of these obese patients were very low, with mean values around 6.3 $\mathrm{mg} / \mathrm{dl}$. We think that the difference in results between our data and the data of Kostner et al. may result from the difference in original $\mathrm{Lp}(\mathrm{a})$ levels, and not due to species difference.

The Lp(a) level in the monkey with an originally high level showed a decrease after 4 days of fasting, while a decrease in TC level was observed at Day 7, after 3 days of banana feeding. Thus, the Lp(a) and TC levels showed independent movement.

The decreased Lp(a) level caused by fasting continued to decrease even after banana feeding, but returned to the original level after the monkey chow feeding. The continuous decrease of $\mathrm{Lp}(\mathrm{a})$ even after banana feeding is very interesting. The bananas weighed about $100 \mathrm{~g}$ weight and had $87 \mathrm{kcal}$ each. One babana consists of $87 \%$ water, $1.1 \%$ protein, $0.1 \%$ fat, $0.3 \%$ fiber, $0.9 \%$ ash and $22.6 \%$ carbohydrate [16]. The difference between monkey chow and banana can be found in the nutritional contents of each. These results indicate that the control of Lp(a) is dependent not only on the amount of food consumed, but also by the type of food. Plasma Lp(a) did not change after an egg diet [17], a lacto-ovovegetarian diet [18], or a prudent diet [7]. However, a low-fat highcarbohydrate diet (15\% protein, $20 \%$ fat, and 65\% carbohydrate) leads to elevation of plasma Lp(a) level [19]. Hence, extreme suppression of protein and/or carbohydrate intake may cause a reduction in plasma Lp(a). We are now interested in knowing whether a change from an ordinary to a vegan diet has a decreasing effect similar to bananas on plasma Lp(a) levels.

The plasma Lp(a) level did not decrease after halving the amount of monkey chow fed even in a monkey with an originally high level of $\mathrm{Lp}(\mathrm{a})$. This indicates that Lp(a) may not affected by mild fasting. However, in this preliminary study, statistical significance among the data acquired from three monkeys have not been clarified yet because number of monkeys was not enough for statistical analysis.

\section{Conclusion}

The present study showed that the plasma Lp(a) levels in cynomolgus monkeys show large individual difference similar to humans. Complete fasting for 4 days decreased the plasma Lp(a) level in a monkey with a high level of $\operatorname{Lp}(\mathrm{a})$, and this decrease continued after banana feeding. The $\mathrm{Lp}(\mathrm{a})$ level returned to the original level by feeding normal monkey chow to the monkey. On the other hand, a monkey with a low level of Lp(a) and a monkey being fed half the amount of monkey chow as the other two monkeys showed no effect on Lp(a) levels. 
These results show that high levels of plasma Lp(a) may be decreased by fasting or feeding of certain foods. Therefore, diet may be used as a method of preventing and/or treating high Lp(a) levels. This study indicates that the cynomolgus monkey can be used as an animal model in place of humans for research of plasma Lp(a) metabolism. However, since we used only 3 monkeys, further studies need to be performed to clarify this.

\section{References}

[1] Malaguarnera, M., Vacante, M., Russo, C., Malaguarnera, G., Antic, T., Malaguarnera, L., Bella, R., Pennisi, G., Galvano, F. and Frigiola, A. (2013) Lipoprotein(a) in Cardiovascular Diseases. BioMed Research International, 2013, Article ID: 650989. http://dx.doi.org/10.1155/2013/650989

[2] Utermann, G. (2001) Lipoprotein(a). In: Scriver, C.R., Beaudet, A.L., Sly, W.S. and Valle, D., Eds., The Metabolic and Molecular Bases of Inherited Disease, McGraw-Hill, New York, 2753-2787.

[3] Hikita, H., Shigeta, T., Kojima, K., Oosaka, Y., Hishikari, K., Kawaguchi, N., Nakashima, E., Sugiyama, T., Akiyama, D., Kamiishi, T., Kimura, S., Takahashi, Y., Kuwahara, T., Sato, A., Takahashi, A. and Isobe, M. (2013) Lipoprotein(a) Is an Important Factor to Determine Coronary Artery Plaque Morphology in Patients with Acute Myocardial Infarction. Coronary Artery Disease, 24, 381-385. http://dx.doi.org/10.1097/MCA.0b013e3283622329

[4] Kostner, K.M. and Kostner, G.M. (2005) Therapy of Hyper-Lp(a). In: von Eckardstein, A., Ed., Atherosclerosis: Diet and Drugs, Springer, Heidelberg, 519-536. http://dx.doi.org/10.1007/3-540-27661-0_19

[5] Wang, Y.T., von Zychlinski, A. and McCormick, S.P. (2009) Dimyristoylphosphotidylcholine Induces Conformational Changes in apoB That Lowers Lipoprotein(a). Journal of Lipid Research, 50, 846-853. http://dx.doi.org/10.1194/jlr.M800428-JLR200

[6] Hjeltnes, G., Hollan, I., Førre, Ø., Wiik, A., Lyberg, T., Mikkelsen, K. and Agewall, S. (2013) Serum Levels of Lipoprotein(a) and E-Selectin Are Reduced Rheumatoid Arthritis Patients Treated with Methotrexate or Methotrexate in Combination with TNF- $\alpha$-Inhibitor. Clinical and Experimental Rheumatology, 31, 415-421.

[7] Schultz, O., Oberhauser, F., Saech, J., Rubbert-Roth, A., Hahn, M., Krone, W. and Laudes, M. (2010) Effects of Inhibition of Interleukin-6 Signalling on Insulin Sensitivity and Lipoprotein(a) Levels in Human Subjects with Rheumatoid Diseases. PLoS One, 5, e14328. http://dx.doi.org/10.1371/journal.pone.0014328

[8] Berglund, L. and Ramakrishnan, R. (2004) Lipoprotein(a): An Elusive Cardiovascular Risk Factor. Arteriosclerosis, Thrombosis, and Vascular Biology, 24, 2219-2226. http://dx.doi.org/10.1161/01.ATV.0000144010.55563.63

[9] Loscalzo, J. (1990) Lipoprotein(a). A Unique Risk Factor for Atherothrombotic Disease. Arteriosclerosis, 10, $672-679$. http://dx.doi.org/10.1161/01.ATV.10.5.672

[10] Fan, J., Shimoyamada, H., Sun, H., Marcovina, S., Honda, K. and Watanabe, T. (2001) Transgenic Rabbits Expressing Human Lipoprotein(a) Develop More Extensive Atherosclerotic Lesions in Response to a Cholesterol-Rich Diet. Arteriosclerosis, Thrombosis, and Vascular Biology, 21, 88-94. http://dx.doi.org/10.1161/01.ATV.21.1.88

[11] Kooshki, A., Taleban, F.A., Tabibi, H. and Hedayati, M. (2011) Effects of $\omega-3$ Fatty Acids on Serum Lipids, Lipoprotein(a) and Hematologic Factors in Hemodialysis Patients. Renal Failure, 33, 892-898. http://dx.doi.org/10.3109/0886022X.2011.605536

[12] Fischer, S., Schatz, U. and Julius, U. (2013) Current Standards in Diagnosis and Therapy of Hyperlipoproteinemia. Atherosclerosis Supplements, 14, 15-18. http://dx.doi.org/10.1016/j.atherosclerosissup.2012.10.035

[13] Makino, K., Abe, A., Maeda, S., Noma, A., Kawade, M. and Takenaka, O. (1989) Lipoprotein(a) in Nonhuman Primates Presence and Characteristics of Lp(a) Immunoreactive Materials Using Anti-Human Lp(a) Serum. Atherosclerosis, 78, 81-85. http://dx.doi.org/10.1016/0021-9150(89)90161-5

[14] Vogt, A. and Parhofer, K.G. (2013) The Potential of Mipomersen, an ApoB Synthesis Inhibitor, to Reduce Necessity for LDL-Apheresis in Patients with Heterozygous Familial Hypercholesterolemia and Coronary Artery Disease. Expert Opinion on Pharmacotherapy, 14, 691-697. http://dx.doi.org/10.1517/14656566.2013.779253

[15] Kostner, G.M., Klein, G. and Krempler, F. (1984) Can Serum Lp(a) Concentrations Be Lowered by Drugs and/or Diet? In: Carlson, L.A. and Olsson, A.G., Eds., Treatment of Hyperlipoproteinemia, Raven Press, New York, 151-156.

[16] Resources Council of the Science and Technology Agency (1982) Standard Tables of Food Composition in Japan. 4th Reviseded, The Science and Technology Agency, Tokyo.

[17] Albers, J.J., Adolphson, J.L. and Hazzard, W.R. (1977) Radioimmunoassay of Human Lp(a) Lipoprotein. Journal of Lipid Research, 18, 331-338.

[18] Masarei, J.R., Rouse, I.L., Lynch, W.J., Robertson, K., Vandongen, R. and Beilin, L.J. (1984) Effects of Lacto-Ovo Vegetarian Diet on Serum Concentrations of Cholesterol, Triglyceride, HDL-C, $\mathrm{HDL}_{2}-\mathrm{C}$, HDL- ${ }_{3} \mathrm{C}$, Apoprotein-B and Lp(a). The American Journal of Clinical Nutrition, 40, 468-478.

[19] Faghihnia, N., Tsimikas, S., Miller, E.R., Witztum, J.L. and Krauss, R.M. (2010) Changes in Lipoprotein(a), Oxidized 
Phospholipids and LDL Subclasses with a Low-Fat High-Carbohydrate Diet. Journal of Lipid Research, 51, 3324-3330. http://dx.doi.org/10.1194/jlr.M005769 
Scientific Research Publishing (SCIRP) is one of the largest Open Access journal publishers. It is currently publishing more than 200 open access, online, peer-reviewed journals covering a wide range of academic disciplines. SCIRP serves the worldwide academic communities and contributes to the progress and application of science with its publication.

Other selected journals from SCIRP are listed as below. Submit your manuscript to us via either submit@scirp.org or Online Submission Portal.
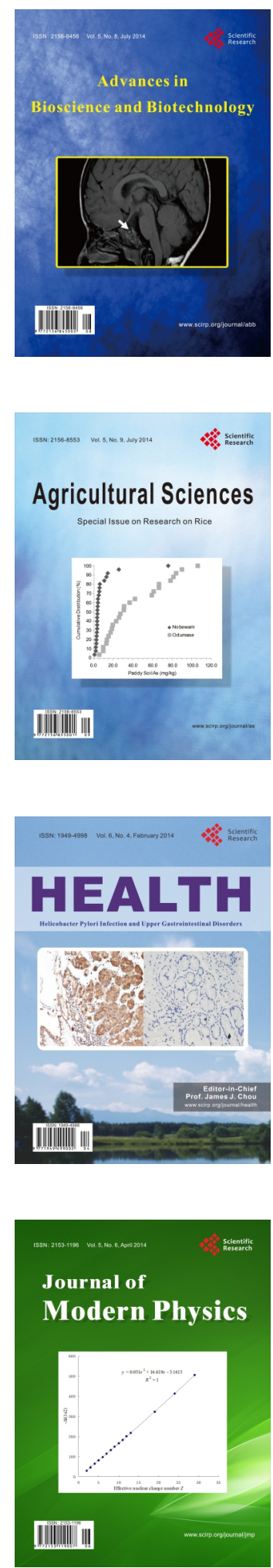
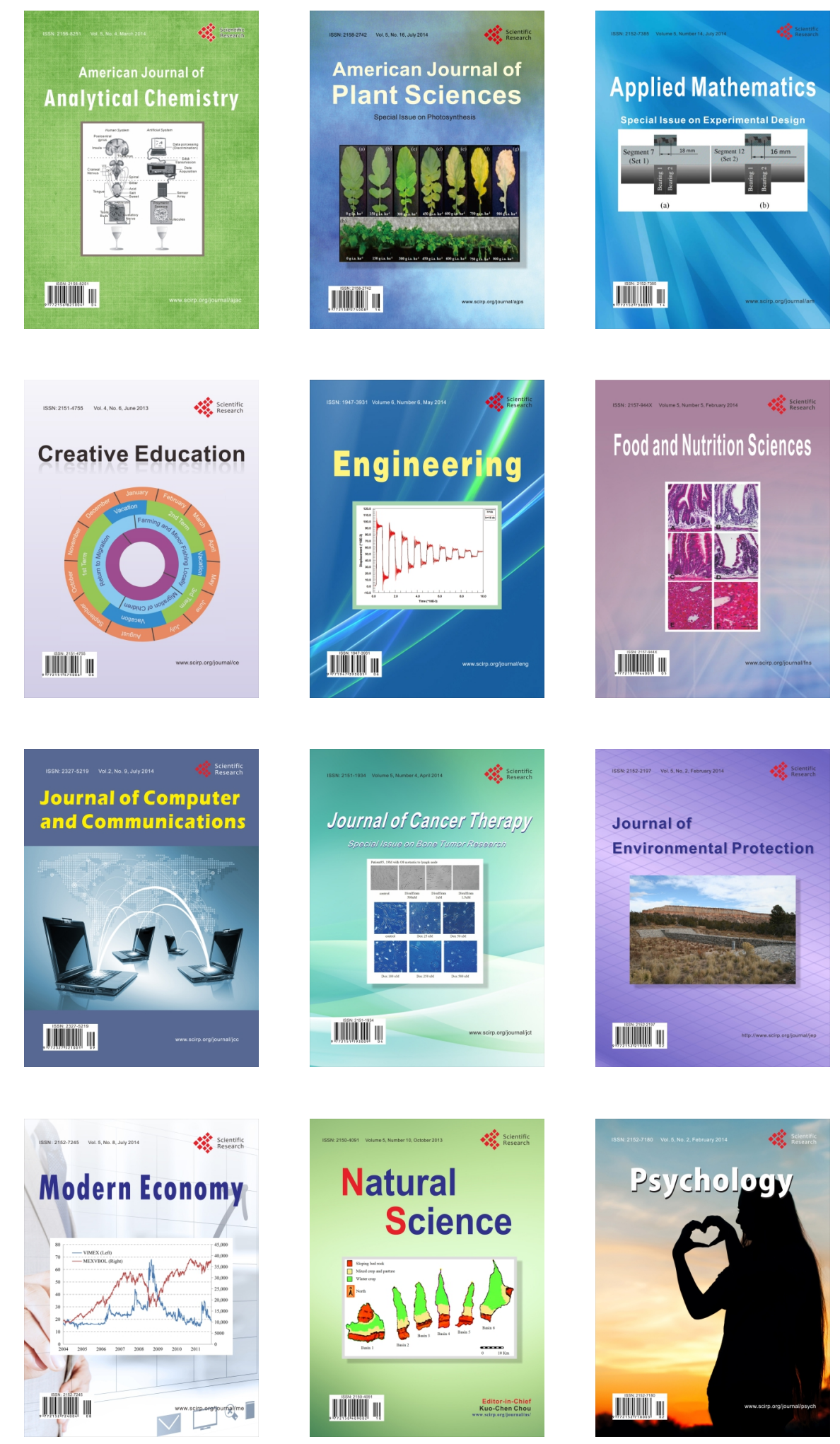